\title{
Notes on unbounded Toeplitz operators in Segal-Bargmann spaces
}

\author{
by D. Cichoń (Kraków)
}

\begin{abstract}
Relations between different extensions of Toeplitz operators $T_{\varphi}$ are studied. Additive properties of closed Toeplitz operators are investigated, in particular necessary and sufficient conditions are given and some applications in case of Toeplitz operators with polynomial symbols are indicated.
\end{abstract}

1. Introduction. In the present paper we continue investigations of Toeplitz operators which has been initiated in [1] and [5] and developed in later works [2], [3] and [4].

Before we present the content of the paper we will introduce some basic notations and definitions.

$A$ is called a linear operator in a Hilbert space $\mathcal{H}$ if its domain $\mathcal{D}(A)$ is a linear subspace in $\mathcal{H}$ and $A: \mathcal{D}(A) \rightarrow \mathcal{H}$ is a linear mapping. We say that a space $\mathcal{D} \subseteq \mathcal{D}(A)$ is a core for $A$ if $\bar{A}=\left(\left.A\right|_{\mathcal{D}}\right)^{-}$.

Consider $L^{2}(\mu)$, the Hilbert space of all complex Borel functions which are square-integrable on $\mathbb{C}^{n}$ with respect to the measure $\mu$ given by the formula $d \mu(z)=\pi^{-n} e^{-\|z\|^{2}} d V(z)$, where $V$ is the Lebesgue measure in $\mathbb{C}^{n}$. The Segal-Bargmann space $\mathcal{B}_{n}$ is a closed subspace in $L^{2}(\mu)$ composed of all analytic functions belonging to $L^{2}(\mu)$. Denote by $\mathcal{E}$ the linear span of the set of functions $e_{a}(z):=e^{\langle z, a\rangle}, a, z \in \mathbb{C}^{n}$, where $\langle\cdot, \cdot\rangle$ is the standard inner product in $\mathbb{C}^{n}$. It can be checked that $f(z)=\left(f, e_{z}\right), f \in \mathcal{B}_{n}, z \in \mathbb{C}^{n}$, so $\mathcal{B}_{n}$ is a Hilbert space with a reproducing kernel. By $\mathcal{P}$ we denote the space of polynomials in $\mathbb{C}^{n}$. Both $\mathcal{P}$ and $\mathcal{E}$ are dense subsets of $\mathcal{B}_{n}$. The sequence $f_{k}(z):=z^{k} / \sqrt{k !}, k \in \mathbb{N}^{n}, z \in \mathbb{C}^{n}$, forms an orthonormal basis for $\mathcal{B}_{n}$, where according to the standard multiindex notation $z^{k}:=z_{1}^{k_{1}} \ldots z_{n}^{k_{n}}$,

1991 Mathematics Subject Classification: Primary 47B35; Secondary 46E20.

Key words and phrases: unbounded operators, Hilbert spaces of entire functions, reproducing kernels.

Research supported by KBN under grant no. PO3A 04110. 
$k !:=k_{1} ! \ldots k_{n}$ ! and $|k|:=\left|k_{1}\right|+\ldots+\left|k_{n}\right|$ for any $z=\left(z_{1}, \ldots, z_{n}\right) \in \mathbb{C}^{n}$ and $k=\left(k_{1}, \ldots, k_{n}\right) \in \mathbb{N}^{n}(\mathbb{N}=\{0,1,2, \ldots\})$.

Let $\varphi: \mathbb{C}^{n} \rightarrow \mathbb{C}$ be a Borel function and $M_{\varphi}$ be the operator of multiplication by $\varphi$ defined in $L^{2}(\mu), \mathcal{D}\left(M_{\varphi}\right):=\left\{f \in L^{2}(\mu): \varphi f \in L^{2}(\mu)\right\}$. Denote by $P$ the orthogonal projection of $L^{2}(\mu)$ onto $\mathcal{B}_{n}$. The Toeplitz operator with symbol $\varphi$ defined in $\mathcal{B}_{n}$ is the operator $T_{\varphi}$ such that $\mathcal{D}\left(T_{\varphi}\right)=\mathcal{D}\left(M_{\varphi}\right) \cap \mathcal{B}_{n}$ and $T_{\varphi} f=P M_{\varphi} f$.

Recall the definitions of the related Toeplitz operators $\Pi_{\varphi}$ and $\widetilde{T}_{\varphi}$. The domain of $\Pi_{\varphi}$ consists of all $f \in \mathcal{B}_{n}$ for which there exists $h \in \mathcal{B}_{n}$ such that $\int_{\mathbb{C}^{n}}(\varphi f-h) \bar{g} d \mu=0$ for all $g \in \mathcal{E}$; then we put $\Pi_{\varphi} f=h$. Taking $\mathcal{P}$ instead of $\mathcal{E}$ in the preceding definition we obtain the definition of $\widetilde{T}_{\varphi}$. It was shown in [3] that $T_{\varphi} \subseteq \Pi_{\varphi} \subseteq \widetilde{T}_{\varphi}$ for any Borel $\varphi$. Considering such extensions of Toeplitz operators is motivated by the fact that they are often more regular than $T_{\varphi}$. The abnormal behaviour of ordinary Toeplitz operators can be illustrated by the remark that it is possible to find a function $\varphi$ such that $T_{\varphi}$ is bounded and densely defined but still $\mathcal{D}\left(T_{\varphi}\right) \varsubsetneqq \mathcal{B}_{n}$.

For a given $p=\sum_{|k|<N} a_{k} f_{k} \in \mathcal{P}$ let us define

$$
p^{*}:=\sum_{|k|<N} \bar{a}_{k} f_{k} \quad \text { and } \quad p(D):=\sum_{|k|<N} a_{k} \frac{\partial^{|k|}}{\partial z_{1}^{k_{1}} \ldots \partial z_{n}^{k_{n}}} .
$$

The following two facts will be useful in the sequel.

Theorem $1.1([5])$. If $p$ is a polynomial in $\mathbb{C}^{n}$ and $f \in \mathcal{D}\left(T_{p}\right)$, then

$$
\|p f\|^{2}=\sum_{j \in \mathbb{N}^{n}} \frac{1}{j !}\left\|\left(D^{j} p^{*}\right)(D) f\right\|^{2} .
$$

Theorem $1.2([3])$. Let $\varphi$ be a Borel function on $\mathbb{C}^{n}$.

(i) If $\mathcal{E} \subseteq \mathcal{D}\left(T_{\varphi}\right)$, then $\left(\left.T_{\varphi}\right|_{\mathcal{E}}\right)^{*}=\Pi_{\bar{\varphi}}=\widetilde{T}_{\bar{\varphi}}$.

(ii) If $\mathcal{P} \subseteq \mathcal{D}\left(T_{\varphi}\right)$, then $\left(\left.T_{\varphi}\right|_{\mathcal{P}}\right)^{*}=\widetilde{T}_{\bar{\varphi}}$.

Let us describe the content of this paper. The main result of the second section is the proof of existence of a Borel function $\varphi$ such that $\mathcal{P} \subseteq \mathcal{D}\left(T_{\varphi}\right)$ and $\Pi_{\varphi} \varsubsetneqq \widetilde{T}_{\varphi}$. The third section is devoted to additive properties of Toeplitz operators. We give necessary and sufficient conditions for the Borel functions $\varphi$ and $\psi$ which guarantee that $T_{\varphi+\psi}=T_{\varphi}+T_{\psi}$. Using these conditions we investigate additive properties of Toeplitz operators with symbols of polynomial type.

2. Relations between $\Pi_{\varphi}$ and $\widetilde{T}_{\varphi}$. It was proved in [4] that $\Pi_{\varphi}=\widetilde{T}_{\varphi}$ in case $\mathcal{E} \subseteq \mathcal{D}\left(\mathcal{T}_{\varphi}\right)$. Below we give a constructive example showing that it is no longer true in general. 
EXAMPLE 2.1. For any non-zero $g \in \mathcal{B}_{n}$, there exists a Borel function $\varphi$ such that $g \in \mathcal{D}\left(\widetilde{T}_{\varphi}\right) \backslash \mathcal{D}\left(\Pi_{\varphi}\right)$.

Proof. For $z \in \mathbb{C}^{n}$ define

$$
\varphi(z):= \begin{cases}\frac{\exp \left(\|z\|^{2}-\left|z_{1}\right|-\ldots-\left|z_{n}\right|\right)}{g(z)}, & g(z) \neq 0 \\ 0, & g(z)=0 .\end{cases}
$$

One can easily check that $\varphi g p \in L^{1}(\mu)$ for all polynomials $p$. Moreover,

$$
\begin{aligned}
\int_{\mathbb{C}^{n}} \varphi(z) g(z) \overline{z^{k}} & d \mu(z) \\
& =\frac{1}{\pi^{n}} \int_{\mathbb{C}^{n}} \overline{z^{k}} e^{-\left|z_{1}\right|-\ldots-\left|z_{n}\right|} d V(z) \\
& =\frac{1}{\pi^{n}} \prod_{j=1}^{n} \int_{0}^{\infty} \int_{0}^{2 \pi} e^{-i k_{j} t_{j}} d t_{j} e^{-r_{j}} r_{j} d r_{j}=0, \quad k \neq(0, \ldots, 0),
\end{aligned}
$$

thus $g \in \mathcal{D}\left(\widetilde{T}_{\varphi}\right)$. Since $\int_{\mathbb{C}^{n}}\left|\varphi g e_{(1, \ldots, 1)}\right| d \mu=\infty$ we conclude that $g \notin$ $\mathcal{D}\left(\Pi_{\varphi}\right)$.

Remark. Taking $g$ identically equal to 1 and defining $\varphi$ as in (2.1) we obtain an example of a polyradially symmetric symbol (i.e. $\varphi(z)=$ $\varphi\left(\left|z_{1}\right|, \ldots,\left|z_{n}\right|\right)$ for almost every $\left.z \in \mathbb{C}^{n}\right)$ for which $\Pi_{\varphi} \varsubsetneqq \widetilde{T}_{\varphi}$. This means that the assumption $\mathcal{P} \subseteq \mathcal{D}\left(T_{\varphi}\right)$ in Theorem 4.1 of [4] cannot be omitted.

Example 2.1 cannot be improved easily to get $\varphi$ such that $\mathcal{P} \subseteq \mathcal{D}\left(T_{\varphi}\right)$. The next theorem insures that such a $\varphi$ exists, but the proof is not constructive.

TheOrem 2.2. There exists a Borel function $\varphi: \mathbb{C}^{n} \rightarrow \mathbb{C}$ such that

(i) $\mathcal{P} \subseteq \mathcal{D}\left(T_{\varphi}\right)$,

(ii) $\Pi_{\varphi} \varsubsetneqq \widetilde{T}_{\varphi}$.

Proof. Assume $n=1$. First we show that the set

$$
\left(\bigcap_{k=0}^{\infty} \mathcal{D}\left(T_{z^{k}}\right)\right) \backslash \mathcal{D}\left(T_{e_{w}}\right)
$$

is not empty in case $w \neq 0$. Suppose that it is empty for some $w \neq 0$. The space $\bigcap_{k=0}^{\infty} \mathcal{D}\left(T_{z^{k}}\right)$ equipped with the distance

$$
d(f, g):=\sum_{k=0}^{\infty} \frac{p_{k}(f-g)}{2^{k}\left(p_{k}(f-g)+1\right)}, \quad \text { where } \quad p_{k}(f):=\|f\|+\left\|T_{z^{k}} f\right\|,
$$

is a metric linear space which is complete because of the completeness of $\mathcal{D}\left(T_{z^{k}}\right)$ with the norm $p_{k}$ for every $k$ in $\mathbb{N}$. Also $\mathcal{D}\left(T_{e_{w}}\right)$ with the graph norm 
$\|f\|_{T_{e_{w}}}=\|f\|+\left\|T_{e_{w}} f\right\|$ is complete. Therefore the linear mapping

$$
\bigcap_{k=0}^{\infty} \mathcal{D}\left(T_{z^{k}}\right) \ni f \rightarrow f \in \mathcal{D}\left(T_{e_{w}}\right)
$$

is closed between complete metric linear spaces. By the closed graph theorem it is continuous, which means that there exist $M>0$ and $s \in \mathbb{N}$ such that

$$
\|f\|+\left\|T_{e_{w}} f\right\| \leq M\left(p_{0}(f)+\ldots+p_{s}(f)\right)
$$

for every $f \in \bigcap_{k=0}^{\infty} \mathcal{D}\left(T_{z^{k}}\right)$. Consider $f=e_{z}, z \in \mathbb{C}$. Then we have $\|f\|=$ $e^{|z|^{2} / 2},\left\|T_{e_{w}} f\right\|=e^{|w+z|^{2} / 2}$ and $p_{j}(f)=\left(\sqrt{q_{j}(|z|)}+1\right) e^{|z|^{2} / 2}(j=0, \ldots, s)$, where $q_{j}$ are some polynomials with nonnegative coefficients, given by Theorem 1.1. Then it follows from (2.3) that

$$
e^{\left(|z+w|^{2}-|z|^{2}\right) / 2} \leq M\left(s+1+\sum_{j=0}^{s} \sqrt{q_{j}(|z|)}\right)
$$

for every $z \in \mathbb{C}$, which is impossible if $w \neq 0$. This contradiction proves that (2.2) is not empty for $w \neq 0$.

Assume $f \in \bigcap_{k=0}^{\infty} \mathcal{D}\left(T_{z^{k}}\right) \backslash \mathcal{D}\left(T_{e_{w}}\right)(w \neq 0)$. Then the function

$$
\varphi(z)= \begin{cases}\bar{f}^{2}(z) / f(z), & f(z) \neq 0 \\ 0, & f(z)=0\end{cases}
$$

satisfies conditions (i) and (ii). Indeed, since $|\varphi(z)|=|f(z)|$ for almost every $z \in \mathbb{C}$ (with respect to $\mu$ ) we easily deduce that $\mathcal{P} \subseteq \mathcal{D}\left(T_{\varphi}\right)$. For the same reason $\varphi f e_{2 w} \notin L^{1}(\mu)$ and consequently $f \notin \mathcal{D}\left(\Pi_{\varphi}\right)$. It remains to show that $f \in \mathcal{D}\left(\widetilde{T}_{\varphi}\right)$. For $j \geq 1$ we have

$$
\int_{\mathbb{C}} \varphi(z) f(z) \bar{z}^{j} d \mu(z)=\int_{\mathbb{C}} \bar{f}^{2}(z) \bar{z}^{j} d \mu(z)=\lim _{R \rightarrow \infty} \overline{\int_{\Delta_{R}} f^{2}(z) z^{j} d \mu(z)}=0,
$$

where $\Delta_{R}:=\{z \in \mathbb{C}:|z|<R\}$. It follows easily that $f \in \mathcal{D}\left(\widetilde{T}_{\varphi}\right)$.

Now we consider the case of $n>1$. Let $f, \varphi$ be as in the one-dimensional case. Define $g(z):=f\left(z_{1}\right)$ and $\psi(z):=\varphi\left(z_{1}\right)$ for all $z \in \mathbb{C}^{n}$. Using the Fubini theorem we get $\mathcal{P} \subseteq \mathcal{D}\left(T_{\psi}\right)$ and $g \in \mathcal{D}\left(\widetilde{T}_{\psi}\right) \backslash \mathcal{D}\left(\Pi_{\psi}\right)$. The proof is complete.

The preceding theorem is related to Theorem 1.3(i) of [4] which states that $\widetilde{T}_{\varphi}=\Pi_{\varphi}$ for every $\varphi$ satisfying $\mathcal{E} \subseteq \mathcal{D}\left(T_{\varphi}\right)$. Thus we have shown that the last condition cannot be replaced by $\mathcal{P} \subseteq \mathcal{D}\left(T_{\varphi}\right)$.

3. Additive properties of Toeplitz operators. In this section we consider conditions on Borel functions $\varphi$ and $\psi$ which guarantee that $T_{\varphi}+$ $T_{\psi}=T_{\varphi+\psi}$. Now we give a criterion which will be applied several times in the sequel. 
Proposition 3.1. Let $\varphi, \psi$ be Borel functions defined on $\mathbb{C}^{n}$. Suppose that $T_{\varphi}, T_{\psi}$ and $T_{\varphi+\psi}$ are closed. Then the following conditions are equivalent:

(i) $T_{\varphi}+T_{\psi}=T_{\varphi+\psi}$.

(ii) $\mathcal{D}\left(T_{\varphi}\right) \cap \mathcal{D}\left(T_{\psi}\right)$ is dense in $\mathcal{D}\left(T_{\varphi+\psi}\right)$ with respect to the graph norm and there exists a constant $C>0$ such that

$$
\left\|T_{\varphi} f\right\|^{2}+\left\|T_{\psi} f\right\|^{2} \leq C\left(\|f\|^{2}+\left\|T_{\varphi+\psi} f\right\|^{2}\right), \quad f \in \mathcal{D}\left(T_{\varphi}\right) \cap \mathcal{D}\left(T_{\psi}\right) .
$$

(iii) $\mathcal{D}\left(T_{\varphi}\right) \cap \mathcal{D}\left(T_{\psi}\right)$ is dense in $\mathcal{D}\left(T_{\varphi+\psi}\right)$ with respect to the graph norm and there exists a constant $C>0$ such that

$$
\|\varphi f\|^{2}+\|\psi f\|^{2} \leq C\left(\|f\|^{2}+\|(\varphi+\psi) f\|^{2}\right), \quad f \in \mathcal{D}\left(T_{\varphi}\right) \cap \mathcal{D}\left(T_{\psi}\right) .
$$

Pro of. Assume that (i) is true. Then $\mathcal{D}\left(T_{\varphi}\right) \cap \mathcal{D}\left(T_{\psi}\right)=\mathcal{D}\left(T_{\varphi+\psi}\right)$ and the mapping

$$
\mathcal{D}\left(T_{\varphi+\psi}\right) \ni f \rightarrow f \in \mathcal{D}\left(T_{\varphi}\right) \cap \mathcal{D}\left(T_{\psi}\right)
$$

is a well defined linear operator acting between complete normed spaces; the norm in $\mathcal{D}\left(T_{\varphi}\right) \cap \mathcal{D}\left(T_{\psi}\right)$ is given by the formula $\|f\|_{1}^{2}=\|f\|^{2}+\left\|T_{\varphi} f\right\|^{2}+$ $\left\|T_{\psi} f\right\|^{2}$, while the topology in $\mathcal{D}\left(T_{\varphi+\psi}\right)$ is given by the graph norm. The mapping (3.3) is closed and by the closed graph theorem we deduce (3.1). We have proved that (ii) holds.

Now assume (ii). Then the mapping

$$
\mathcal{D}_{1} \ni f \rightarrow f \in \mathcal{D}_{2}
$$

is continuous, where $\mathcal{D}_{1}=\mathcal{D}\left(T_{\varphi}\right) \cap \mathcal{D}\left(T_{\psi}\right)$ with the graph norm induced from $\mathcal{D}\left(T_{\varphi+\psi}\right)$, and $\mathcal{D}_{2}=\mathcal{D}\left(T_{\varphi}\right) \cap \mathcal{D}\left(T_{\psi}\right)$ but with the norm $\|\cdot\|_{1}$ defined above. Since $\mathcal{D}_{1}$ is dense in $\mathcal{D}\left(T_{\varphi+\psi}\right)$ it is possible to extend the mapping (3.4) to the whole space $\mathcal{D}\left(T_{\varphi+\psi}\right)$. This implies the inclusion $\mathcal{D}\left(T_{\varphi+\psi}\right) \subseteq \mathcal{D}\left(T_{\varphi}\right) \cap \mathcal{D}\left(T_{\psi}\right)$.

The equivalence of (ii) and (iii) is an immediate consequence of Proposition 2.3 of [4].

Although the assumption of the closedness of Toeplitz operators may seem to be restrictive, the preceding proposition may be used in many cases, for instance when $\varphi$ and $\psi$ are analytic. The following proposition shows that in the multidimensional case Toeplitz operators do not have good additive properties even for the very simple choice of $\varphi$ and $\psi$.

In what follows $(p \otimes 1)(z, w)=p(z)$ and $(1 \otimes q)(z, w)=q(w)$, where $z \in \mathbb{C}^{n}, w \in \mathbb{C}^{m}$.

Proposition 3.2. Let $p, q$ be nonconstant polynomials in $\mathbb{C}^{n}$ and $\mathbb{C}^{m}$ respectively. Then

$$
T_{p \otimes 1}+T_{1 \otimes q} \varsubsetneqq T_{p \otimes 1+1 \otimes q} .
$$


Pr o of. Assume that $T_{p \otimes 1}+T_{1 \otimes q}=T_{p \otimes 1+1 \otimes q}$. Applying Proposition 3.1 we conclude that there exists a constant $C>0$ such that

$$
\begin{array}{r}
\|(p \otimes 1) f\|^{2}+\|(1 \otimes q) f\|^{2} \leq C\left(\|f\|^{2}+\|(p \otimes 1+1 \otimes q) f\|^{2}\right), \\
f \in \mathcal{D}\left(T_{p \otimes 1}\right) \cap \mathcal{D}\left(T_{1 \otimes q}\right) .
\end{array}
$$

Consider $f=e_{a}$ for $a=\left(a_{1}, a_{2}\right), a_{1} \in \mathbb{C}^{n}, a_{2} \in \mathbb{C}^{m}$. Theorem 1.1 implies that

$$
\begin{aligned}
\left\|(p \otimes 1) e_{a}\right\|^{2} & =\sum_{j \in \mathbb{N}^{n}} \frac{1}{j !}\left|\left(D^{j} p\right)\left(a_{1}\right)\right|^{2} e^{\|a\|^{2}} \\
\left\|(1 \otimes q) e_{a}\right\|^{2}= & \sum_{j \in \mathbb{N}^{m}} \frac{1}{j !}\left|\left(D^{j} q\right)\left(a_{2}\right)\right|^{2} e^{\|a\|^{2}} \\
\left\|(p \otimes 1+1 \otimes q) e_{a}\right\|^{2}= & \left|p\left(a_{1}\right)+q\left(a_{2}\right)\right|^{2} e^{\|a\|^{2}}+\sum_{j \in \mathbb{N}^{n} \backslash\{0\}} \frac{1}{j !}\left|\left(D^{j} p\right)\left(a_{1}\right)\right|^{2} e^{\|a\|^{2}} \\
& +\sum_{j \in \mathbb{N}^{m} \backslash\{0\}} \frac{1}{j !}\left|\left(D^{j} q\right)\left(a_{2}\right)\right|^{2} e^{\|a\|^{2}} .
\end{aligned}
$$

Now inequality (3.6) can be rewritten as follows:

$$
\begin{array}{r}
\left|p\left(a_{1}\right)\right|^{2}+\left|q\left(a_{2}\right)\right|^{2}+r\left(a_{1}, a_{2}\right) \leq C\left(1+r\left(a_{1}, a_{2}\right)+\left|p\left(a_{1}\right)+q\left(a_{2}\right)\right|^{2}\right), \\
a_{1} \in \mathbb{C}^{n}, a_{2} \in \mathbb{C}^{m},
\end{array}
$$

where

$$
r\left(a_{1}, a_{2}\right):=\sum_{j \in \mathbb{N}^{n} \backslash\{0\}} \frac{1}{j !}\left|\left(D^{j} p\right)\left(a_{1}\right)\right|^{2}+\sum_{j \in \mathbb{N}^{m} \backslash\{0\}} \frac{1}{j !}\left|\left(D^{j} q\right)\left(a_{2}\right)\right|^{2} .
$$

It is possible to choose $a_{1} \in \mathbb{C}^{n}$ and $a_{2} \in \mathbb{C}^{m}$ such that the polynomial $\widetilde{p}(x):=p\left(x a_{1}\right), x \in \mathbb{C}$, (resp. $\left.\widetilde{q}(y):=q\left(y a_{2}\right), y \in \mathbb{C}\right)$ has the same degree as $p$ (resp. q). Define also $\widetilde{r}(x, y):=r\left(x a_{1}, y a_{2}\right)$ for $x, y \in \mathbb{C}$. Then

$$
\begin{aligned}
|\widetilde{p}(x)|^{2}+|\widetilde{q}(y)|^{2}+ & \widetilde{r}(x, y) \\
& \leq C\left(1+\widetilde{r}(x, y)+|\widetilde{p}(x)+\widetilde{q}(y)|^{2}\right), \quad x, y \in \mathbb{C} .
\end{aligned}
$$

Using the fact that

$$
\frac{\widetilde{r}(x, y)}{|x|^{2 \operatorname{deg} p}+|y|^{2 \operatorname{deg} q}} \rightarrow 0, \quad|x| \rightarrow \infty,|y| \rightarrow \infty,
$$

we deduce that (3.7) is impossible since one can find two sequences $\left\{x_{k}\right\}$ and $\left\{y_{k}\right\}$ tending to infinity such that $\left|\widetilde{p}\left(x_{k}\right)+\widetilde{q}\left(y_{k}\right)\right|=0$.

Now we consider the one-dimensional case. The next proposition will be helpful in order to obtain examples of symbols for which the additive property of Toeplitz operators holds and which are different from those included in [4, Theorem 3.3]. 
Proposition 3.3. If $p$ and $q$ are polynomials in $\mathbb{C}$ such that $\left|\left(d^{N} p / d z^{N}\right)(1)\right| \neq\left|\left(d^{N} q / d z^{N}\right)(1)\right|$, where $N:=\max \{\operatorname{deg} p, \operatorname{deg} q\}$, then $\mathcal{P}$ is a core for $T_{p+\bar{q}}$ and $T_{p+\bar{q}}^{*}=\widetilde{T}_{\bar{p}+q}=\Pi_{\bar{p}+q}$.

It turns out that the assumption $\left|\left(d^{N} p / d z^{N}\right)(1)\right| \neq\left|\left(d^{N} q / d z^{N}\right)(1)\right|$ is superfluous [6], nevertheless we will use it in the proof of our main result (Theorem 3.4).

Pr o of. The main step of the proof is to show that $\mathcal{D}\left(T_{\psi}\right)=\mathcal{D}\left(T_{p+\bar{q}}\right)$ for $\psi(z):=\max _{|\lambda|=1}|p(\lambda z)+\bar{q}(\lambda z)|, z \in \mathbb{C}$. Obviously $\mathcal{D}\left(T_{\psi}\right) \subseteq \mathcal{D}\left(T_{p+\bar{q}}\right)$. For the opposite inclusion it suffices to prove that there are constants $R, c, d>0$ such that

$$
\psi(z) \leq c+d|p(z)+\bar{q}(z)|
$$

for every $z \in \mathbb{C}$ satisfying $|z|>R$. But (3.8) follows from the inequalities

$$
\begin{gathered}
|p(z)+\bar{q}(z)| \geq \frac{1}{2 N !}|| \frac{d^{N} p}{d z^{N}}(1)|-| \frac{d^{N} q}{d z^{N}}(1)|| \cdot\left|z^{N}\right|, \\
\psi(z) \leq\left(\frac{1}{N !}\left|\frac{d^{N} p}{d z^{N}}(1)\right|+\frac{1}{N !}\left|\frac{d^{N} q}{d z^{N}}(1)\right|+1\right)\left|z^{N}\right|,
\end{gathered}
$$

which are valid for $|z|$ sufficiently large. We have proved that $\mathcal{D}\left(T_{\psi}\right)=$ $\mathcal{D}\left(T_{p+\bar{q}}\right)$. In fact, we obtained the following property for the function $p+\bar{q}$ : there are constants $R, c, d>0$ such that $\left|p\left(e^{i t} z\right)+\bar{q}\left(e^{i t} z\right)\right| \leq c+d|p(z)+\bar{q}(z)|$ for $|z|>R$ and $t \in \mathbb{R}$. This enables us to apply Theorem 1.3. of [4] to get $\left(\left.T_{p+\bar{q}}\right|_{\mathcal{P}}\right)^{-}=\bar{T}_{p+\bar{q}}$. The proof of this theorem is based on a theorem of Berezin [1] on weighted polynomial approximation.

However, it is possible to find a direct argument, which is presented below. Since the graph norm induced by $T_{p+\bar{q}}$ is dominated by the graph norm of the operator $\left.M_{\psi}\right|_{\mathcal{D}\left(T_{\psi}\right)}$ it remains to check that the space of polynomials is dense in $\mathcal{D}\left(T_{\psi}\right)$ with respect to the norm $\|\cdot\|_{1}$ given by the formula $\|h\|_{1}^{2}=\|h\|^{2}+\left\|M_{\psi} h\right\|^{2}, h \in \mathcal{D}\left(T_{\psi}\right)$. Suppose that $f=\sum_{k=0}^{\infty} a_{k} f_{k} \in \mathcal{D}\left(T_{\psi}\right)$ is orthogonal to $\mathcal{P}$ with respect to the inner product which induces the norm $\|\cdot\|_{1}$. We have

$$
\begin{aligned}
0 & =\left(f, f_{s}\right)+\left(\psi f, \psi f_{s}\right)=\int_{\mathbb{C}} f \bar{f}_{s}\left(1+\psi^{2}\right) d \mu \\
& =\frac{1}{\pi \sqrt{s !}} \int_{0}^{\infty} \int_{0}^{2 \pi} f\left(r e^{i t}\right) e^{-i s t} d t r^{s+1}\left(1+\psi^{2}(r)\right) e^{-r^{2}} d r \\
& =\frac{2 a_{s}}{s !} \int_{0}^{\infty} r^{2 s+1}\left(1+\psi^{2}(r)\right) e^{-r^{2}} d r
\end{aligned}
$$

for any $s \in \mathbb{N}$, hence $a_{s}=0$ for all $s \in \mathbb{N}$ and $f=0$. The equalities $T_{p+\bar{q}}^{*}=\widetilde{T}_{\bar{p}+q}=\Pi_{\bar{p}+q}$ follow from Theorem 1.2. 
The next theorem gives examples of Toeplitz operators having the additivity property.

Theorem 3.4. If $\alpha \in \mathbb{C},|\alpha|>1$, and $p$ is a polynomial in $\mathbb{C}$, then $\left(\left.T_{\alpha p+\bar{p}}\right|_{\mathcal{P}}\right)^{-}=T_{\alpha p+\bar{p}}$ and $T_{\alpha p+\bar{p}}=\alpha T_{p}+T_{\bar{p}}$.

Pr o of. To prove the first equality it suffices to check the closedness of the operator $T_{\alpha p+\bar{p}}$ and apply Proposition 3.3. According to the criterion of closedness of Toeplitz operators proved in [4, Proposition 2.3] for a given $\alpha$ we will find a constant $C>0$ such that

$$
\|(\alpha p+\bar{p}) r\|^{2} \leq C\left(\|r\|^{2}+\left\|T_{\alpha p+\bar{p}} r\right\|^{2}\right), \quad r \in \mathcal{P} .
$$

From the identities

$$
\begin{aligned}
\|(\alpha p+\bar{p}) r\|^{2} & =\left(|\alpha|^{2}+1\right)\|p r\|^{2}+2 \operatorname{Re}(\alpha p r, \bar{p} r), \\
\left\|T_{\alpha p+\bar{p}} r\right\|^{2} & =|\alpha|^{2}\|p r\|^{2}+\|P(\bar{p} r)\|^{2}+2 \operatorname{Re}(\alpha p r, \bar{p} r)
\end{aligned}
$$

one can deduce that it suffices to show that for any $|\alpha|>1$ there exists $C>0$ such that

$$
\begin{aligned}
& \left(C|\alpha|^{2}-|\alpha|^{2}-1\right)\|p r\|^{2} \\
& \quad-|2-2 C| \cdot|\alpha| \cdot\|p r\| \cdot\|P(\bar{p} r)\|+C\|P(\bar{p} r)\|^{2} \geq 0, \quad r \in \mathcal{P} .
\end{aligned}
$$

This in turn follows from the fact that for every $|\alpha|>1$ one can find $C>1$ such that the function

$$
G(x, y)=\left(C|\alpha|^{2}-|\alpha|^{2}-1\right) x^{2}+(2-2 C)|\alpha| x y+C y^{2}
$$

is nonnegative for $x, y \in \mathbb{R}, x \geq y \geq 0$. The proof of (3.10) is left to the reader. Hence we have proved that $T_{\alpha p+\bar{p}}$ is closed.

In order to prove that $T_{\alpha p+\bar{p}}=\alpha T_{p}+T_{\bar{p}}$ we will use Proposition 3.1. The space $\mathcal{D}\left(T_{p}\right) \cap \mathcal{D}\left(T_{\bar{p}}\right)$ is dense in $\mathcal{D}\left(T_{\alpha p+\bar{p}}\right)$ because the polynomials form a core for $T_{\alpha p+\bar{p}}$. The inequality

$$
|| \alpha|-1| \cdot\|p h\| \leq\|(\alpha p+\bar{p}) h\|, \quad h \in \mathcal{D}\left(T_{p}\right),
$$

implies (3.2) of Proposition 3.1(iii), and this completes the proof.

Remark. It is necessary to assume $|\alpha| \neq 1$ in Theorem 3.4 because $T_{p+\bar{p}}$ is not always closed (cf. [4, Example 6.2]).

The last proposition is strictly related to [4, Theorem 3.3]. It is shown that in general it suffices to take polynomials of different degrees to obtain another example of Toeplitz operators with the additivity property.

Proposition 3.5. If $p$ and $q$ are polynomials in one complex variable such that $\operatorname{deg} p \neq \operatorname{deg} q$, then $\left(\left.T_{p+\bar{q}}\right|_{\mathcal{P}}\right)^{-}=\bar{T}_{p+\bar{q}}$ and $T_{p+\bar{q}}=T_{p}+T_{\bar{q}}$.

Proof. The following fact was proved in [4, Theorem 3.3]: if $p$ and $q$ are polynomials in $\mathbb{C}$ such that $\operatorname{deg} p<\operatorname{deg} q=N$, then $\mathcal{D}\left(\widetilde{T}_{p+\bar{q}}\right) \subseteq \mathcal{D}\left(T_{z^{N}}\right)$. From this one can easily deduce that $\mathcal{D}\left(T_{p+\bar{q}}\right) \subseteq \mathcal{D}\left(T_{z^{N}}\right)$, where $p$ and $q$ 
are polynomials of different degrees and $N=\max \{\operatorname{deg} p, \operatorname{deg} q\}$. Therefore $\mathcal{D}\left(T_{p+\bar{q}}\right)=\mathcal{D}\left(T_{p}\right) \cap \mathcal{D}\left(T_{\bar{q}}\right)=\mathcal{D}\left(T_{z^{N}}\right)$, which implies the second equality.

Proposition 3.3 implies that $\mathcal{P}$ is a core for $T_{p+\bar{q}}$, but we give an alternative proof based on the fact mentioned above. Suppose that $p=\sum_{j=0}^{N} p_{j} f_{j}$ and $q=\sum_{j=0}^{M} q_{j} f_{j}, \operatorname{deg} p \neq \operatorname{deg} q$. Then it follows that

$$
T_{p+\bar{q}}=\sum_{j=0}^{N} p_{j} T_{f_{j}}+\sum_{j=0}^{M} q_{j} T_{\bar{f}_{j}} .
$$

Given $g=\sum_{k=0}^{\infty} a_{k} f_{k} \in \mathcal{D}\left(T_{p+\bar{q}}\right)$, set $g^{(N)}:=\sum_{k=0}^{N} a_{k} f_{k}$. Since $g^{(N)} \rightarrow g$, $T_{f_{j}} g^{(N)} \rightarrow T_{f_{j}} g$ and $T_{\bar{f}_{j}} g^{(N)} \rightarrow T_{\bar{f}_{j}} g, j \in \mathbb{N}$, we deduce that $\mathcal{P}$ is a core for $T_{p+\bar{q}}$. The proof is complete.

\section{References}

[1] F. A. Berezin, Vick and anti-Vick symbols of operators, Mat. Sb. 86 (1971), 578-610 (in Russian).

[2] J. Janas, Toeplitz and Hankel operators on Bargmann spaces, Glasgow Math. J. 30 (1988), 315-323.

[3] - Unbounded Toeplitz operators in the Bargmann-Segal space, Studia Math. 99 (1991), 87-99.

[4] J. Janas and J. Stochel, Unbounded Toeplitz operators in the Segal-Bargmann space. II, J. Funct. Anal. 126 (1994), 418-447.

[5] D. J. Newman and H. S. Shapiro, Fischer spaces of entire functions, in: Entire Functions and Related Parts of Analysis, J. Korevaar (ed.), Proc. Sympos. Pure Math. 11, Amer. Math. Soc., Providence, 1968, 360-369.

[6] J. Jan as and J. Stochel, private communication.

Institute of Mathematics

Jagiellonian University

Reymonta 4

30-059 Kraków, Poland

E-mail: cichon@im.uj.edu.pl

Reçu par la Rédaction le 18.5.1995

Révisé le 20.12.1995 